\title{
Strategy of Third-Party Automotive Logistics Distribution Network with Random Demand
}

\author{
Qin Lü, a , Su Ying ${ }^{2, b}$ \\ ${ }^{1}$ School of traffic and transportation, Beijing jiaotong University, Beijing, China \\ ${ }^{2}$ School of traffic and transportation, Beijing jiaotong University, Beijing, China \\ a qinlu56@126.com, b15120873@bjtu.edu.cn
}

\begin{abstract}
Keywords: Distribution Network, 3PL, Automotive Logistics
Abstract. In this paper, we addressed the inventory-location model of the distribution network planning based on the actual vehicle 3PL case background. We use the genetic algorithm to calculate the actual data, and evaluate the effect from the demand and cost two aspects. The model deter-mines the number and location of required warehouses, the inventory level at each retailer. We analyze the current domestic automobile logistics policy and involved random variables in the mathematic part.
\end{abstract}

\section{Introduction}

the automotive industry chain is facing more intense market competition. Consumers not only concerned about the performance and quality of the vehicle they bought, but also show higher requirements of supporting services. The market decentralization caused the diversification of the market and increased the uncertainty of demand. The current p-to-p mode could avoid the need of midway handling, so to a certain extent, reduce the risk of occurrence of product damage. However, while the dealers are located all over the country, the waiting time is influenced significantly by the accuracy of the sales, capacity of trunk, the distance between plants to destinations. This paper focused on reducing the delivery time of orders, and improving the efficiency of existing facilities while reducing inventory costs.

\section{Literature review}

Recently, supply chain managers and researchers have realized the importance of the integration of supply chain decisions. Zhang Mengxia (2012) ${ }^{[3]}$ focused on the research of system randomness when the problem expands to the whole distribution network. To solve the problem of high complexity of large-scale network problem, the inventory model of

two-level network is deduced, and the three-level network problem is verified by empirical calculation to verify its validity. As for the feasibility of 3PL inventory Controlling, Dai Jun, Shei et (2015) ${ }^{[4]}$ analyzed the VMI management mode of the 3PLs, and obtained the conclusion that 3PL owns inventory decision-making power can improve the overall performance of the supply chain. But the model design is more ideal than the actual situation on some aspects.

This research will design a more practical and effective location-inventory model by digging into the actual cases.

\section{Problem Analysis}

This paper discusses the whole vehicle logistics network location-inventory problem with a three-level distribution network system consists of plants, distribution Centers and dealers. Multiple plant distribution network is as shown in Fig.1 


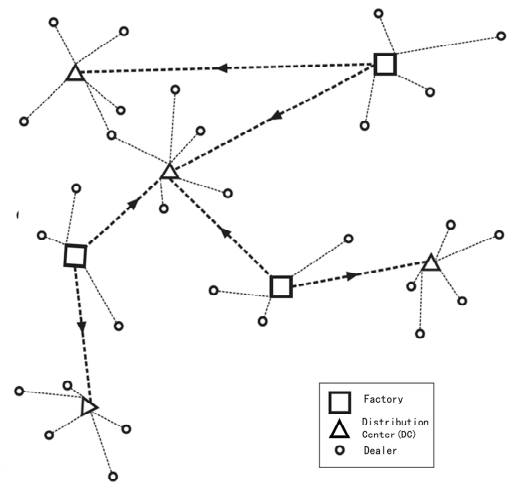

Fig. 1 Inventory-Location Model Network

The main objectives of this model are as follow: (1) to determine the optimal number and location of the distribution centers; (2) to determine the serving areas of each distribution center (3) to determine the optimal replenishment strategy for each distribution center.

In this paper, we select cities with competitive advantages as the alternative collection of distribution centers from the perspective of system economy, implementation convenience and strategic rationality. The optimal location scheme is determined from the set of alternative points by using the model quantitative calculation. The qualitative filtering process is shown in Fig.2

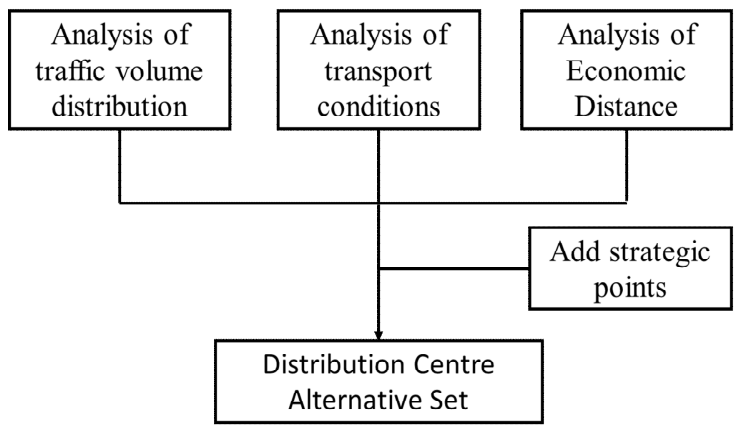

Fig. 2 Alternative DC collection

Assumption. (i) Single-item vehicle is produced by different plants.(ii) The plant, distributor, alternative distribution center location is fixed and known.(iii) The productivity of plants and capacity of DCs are infinite.(iv) The transportation cost of one single vehicle from plant to the distribution center is linearly related to the transportation distance.(v) Each warehouse follows a continuous inventory policy, i.e., fixed quantity $\mathrm{Q}$ is ordered from the plant when the inventory level at the distribution center falls to or below a certain point r.(vi) The shipping time from plant to the DCs obeys the gamma distributions.(vii) Each dealer has an uncertain independent demand that follows a normal distribution with known mean and variance.

Param

Parameter. $i \in I$ :Set of alternative DCs; $j \in J$ :Set of dealers; $l \in L:$ Set of plants; $d_{j l}$ :demand per year for each dealer $j$ from factory $l ; D_{i l}$ :demand per year for each DC $i$ from factory $l$; $O C_{i l}:$ Fixed inventory ordering cost from factory per unit of product per time unit at $\mathrm{DC} i ; Z D C_{i l}$ :Transportation cost rate in main lines; $D R C_{i l}$ : Transportation cost rate in stem lines; $s_{i l}$ :Distribution Center $i$ Product $l$ Safety Inventory; $Q_{i l}$ : Distribution Center $i$ product $l$ replenishment volume; $A I_{i l}$ :Average inventory of product $l$ in distribution center $i ; F_{i}$ : Annual fixed cost per distribution center $i$;HC : Distribution Center storage Cost; $P C_{i}$ : Out of stock cost $X_{i}=\left\{\begin{array}{l}1, \text { if we locat at candidate DC } i \\ 0, \text { if not }\end{array}\right.$ 


$$
Y_{i j l}=\left\{\begin{array}{l}
1, \text { if demands at dealer } i \text { are } \\
\text { assigned to a DC at candidate site } j \\
0, \text { if not }
\end{array}\right.
$$

\section{Inventory Analysis}

Dealer inventory analysis. Assuming that the daily demand of dealer $j$ is independent of the same distribution of random variables, the dealer's demand for the commodity vehicles obeys normal distribution $N\left(\mu_{j l}, \sigma_{j l}^{2}\right)$, Daily demand mean value is $\mu_{j l}$, daily demand variance is $\sigma_{j l}^{2}$, total annual demand is $d_{i l}=\chi \mu_{j l}$, where $\chi$ is constant.

It is out of stock to be unable to reach the distributor within the stipulated time $\eta$ when order. Assuming that the distribution center is out of stock, the waiting time of the distributor to which it serves is the same, with $W T_{i l}$ as the representation.

According to the formula of Little, the average number of individuals in a queueing system is equal to that of an individual and the average length of stay. Therefore, the Dealers' order in the distribution center average shortage of goods equal to the demand arrival rate and order waiting time of the product in the distribution system:

$$
E\left(W T_{i j l}\right)=\frac{E\left(S O_{i l}\right)}{\sum_{j \in J} \mu_{i j l} Y_{i j l}}
$$

Assuming that waiting time at dealers obey the normal distribution and the probability distribution density is $g(t)$, Therefore, the average waiting time of the distributor order at the distribution center is shown in Eq.1.

$$
E\left(W T_{i j l}\right)=\int_{0}^{\infty}\left[1-g\left(\frac{x-\mu_{\omega}}{\sigma_{\omega}}\right)\right] d x
$$

Let $\frac{x-\mu_{\omega}}{\sigma_{\omega}}=t$, when $t_{1}-t_{2} \geq \eta$, Orders are not waiting, so they can be pushed that

$$
\begin{aligned}
& V\left[W T_{i j l}\right]=E\left[W T_{i j l}^{2}\right]-E\left[W T_{i j l}\right]^{2} \\
& =\sigma_{\omega}^{2}\left(1-p\left(W T_{i j l}=0\right)\right)+E\left[W T_{i j l}\right] \cdot \mu_{\omega}-E\left[W T_{i j l}\right]^{2}
\end{aligned}
$$

Shortage of dealer is:

$$
=\sigma_{\omega}^{2} \cdot \Phi\left(-\frac{\mu_{\omega}}{\sigma_{\omega}}\right)+E\left[W T_{i j l}\right] \cdot \mu_{\omega}-E\left[W T_{i j l}\right]^{2}
$$

$$
E S_{j l}=\int_{\eta \cdot \mu_{j l}}^{\infty}\left(x_{j l}-\eta \cdot \mu_{j l}\right) \cdot \varphi\left(x_{j l}\right) d x_{j l}
$$

As a result, the distribution centers are out of stock

$$
E S S_{i}=\sum_{l \in L} \sum_{j \in J} E S_{j l} \cdot Y_{i j l}
$$

Distribution center inventory analysis. The daily demand of distributors is independent and identically distributed stochastic variable, and the demand for each kind of commercial vehicle obeys the normal distribution $N\left(\mu_{j l}, \sigma_{j l}^{2}\right)$. Therefore, the demand for the allocation centers also obeys the normal distribution $N\left(\mu_{i}, \sigma_{i}^{2}\right)$. Total annual demand volume is $D_{i}$.

$$
\mu_{i l}=\sum_{l \in L} \sum_{j \in J} \mu_{j l} Y_{i j l}
$$




$$
\begin{gathered}
\sigma_{i l}^{2}=\sum_{l \in L} \sum_{j \in J} \sigma_{j l}^{2} Y_{i j l} \\
D_{i}=\chi \sum_{j \in J} \mu_{j} Y_{i j}=\chi \mu_{i}
\end{gathered}
$$

Assuming that the demand in the lead time $L T_{i l} D$ obeys the normal distribution, the distribution density function and the distribution function are respectively $\varphi_{i l}(x)$ and $\psi_{i l}(x)$. The mean value and variance of the allocation center demand in its replenishment lead time are as follow:

$$
\begin{gathered}
E\left(L T_{i l} D\right)=\mu_{i l} \cdot E\left(L T_{i l}\right) \\
V\left(L T_{i l} D\right)=\sigma_{i l}^{2} \cdot E\left(L T_{i l}\right)+\mu_{i l}^{2} \cdot V\left(L T_{i l}\right)
\end{gathered}
$$

Objective function.

$$
\min \sum_{i \in I} F_{i} \cdot X_{i}+\sum_{l \in L} \sum_{i \in I} \sum_{j \in J}\left(D R C_{i j}+Z D C_{i l}\right) \cdot \mu_{j l} \cdot Y_{i j l}+\sum_{l \in L} \sum_{i \in I} \sum_{j \in J}\left(\frac{1}{2} \cdot Q_{i l}+Y_{i j l} \cdot s_{i l}\right) \cdot H C_{i l}+\sum_{i \in I} P C_{i} \cdot E S S_{i}
$$

The first part of the Target function indicates the fixed cost of setting up the distribution centers; the second part indicates the transportation cost of the dealers from the factory through the DCs, and the third part indicates the inventory cost; and the last part indicates the Out of stock cost.

\section{Constraint.}

$$
\begin{gathered}
\sum_{j \in J} Y_{i j l}=1, \forall i \in I, j \in J, l \in L \\
Y_{i j l} \leq X_{i}, \forall i \in I, j \in J, l \in L \\
\sum_{j \in J} X_{i} \geq 1, \forall i \in I \\
Q_{i l}=\min \left\{Q_{i l}^{*}, C A Q_{i l}\right\} \\
X_{i} \in\{0,1\}_{i}, \forall i \in I \\
Y_{i j l} \in\{0,1\}_{i}, \forall i \in I, j \in J, l \in L
\end{gathered}
$$

\section{Solution}

Algorithm structure. The problem above is a typical 0-1-integer nonlinear programming problem, with increasing number of plants, distribution centers and dealers. So, this paper will solve the model by genetic algorithm with method shown in Fig.3.

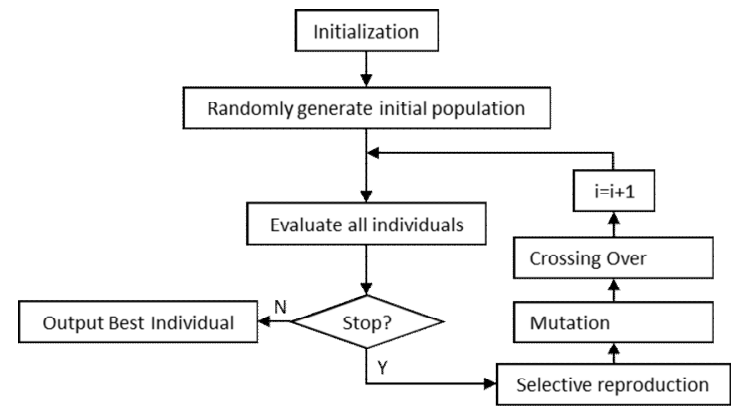

Fig. 3 Genetic algorithm for solving flow diagram

\section{Case Study}

Background. A third-party automotive Logistics Enterprise W company mainly serves three automobile production enterprises whose respective host plants are located in Changchun, Chengdu and Foshan in China and ales coverage nationwide 
In order to improve the efficiency of logistics and to better respond to market changes with lower costs, enterprises W plan to redesign the distribution network. 17 cities are chosen to an alternative set .

Computational results and analysis. Assign the data of $\mathrm{W}$ enterprise's year-round demand, cost and aging generation into the model. The optimal value tracking image of the genetic algorithm after 1000 iterations is shown in Fig.7. The results of final distribution center location are Changchun, Chengdu, Foshan, Beijing, Hefei, Suzhou, Shijiazhuang, Taiyuan, Wuhan, Xi ' an, Zhengzhou.

As shown in Fig.4 to set the total 6 distribution center, the the decline of inventory cost reaches $23 \%$.

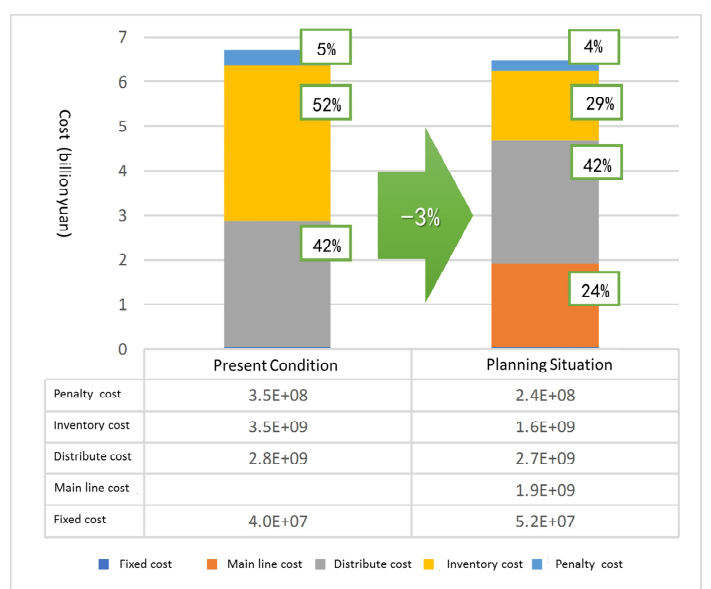

Fig. 4 Distribution network optimization cost composition comparison

As shown in Fig.5, the distribution center inventory turnover average of $1.5 \sim 2.5$ days. Inventory diversification will significantly improve the service level than before which can ensure that more than $90 \%$ orders be delivered within 2 days.

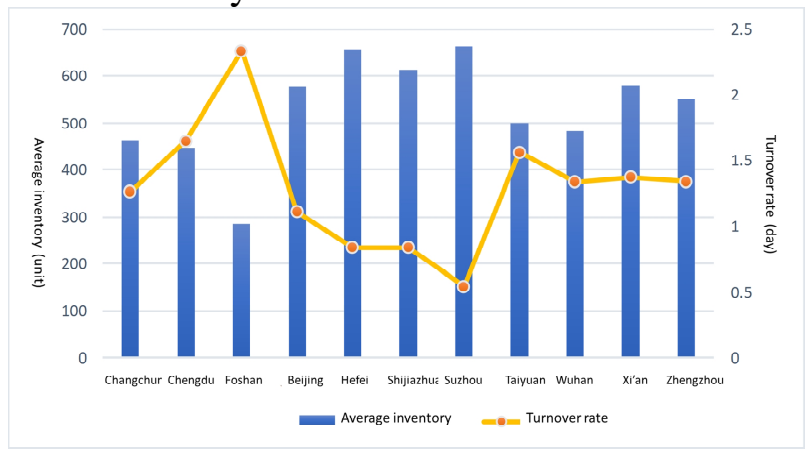

Fig. 5 Average inventory and turnover rate of each

\section{Conclusion}

This paper focus on the objective that the customer stochastic demand of the whole vehicle distribution which obey the normal distribution. Taking 3PL Enterprise Service level, out of stock cost, inventory holding cost into account, we set up a comprehensive Logistics Model, which based on consideration of transport scale economic effect, inventory Control strategy, supply chain logistics costs, Service level and other factors. We used data based on actual case to evaluate the model results. This paper also discussed the influence of 3PL schemes exert to the cost and service level of the whole supply chain. This paper provided the reference basis for the Logistics network optimization and upgrading of the third-party automobile logistics enterprises. 


\section{References}

[1] Li Xinyue. Research on Optimization of logistics system of Volkswagen FAW platform based on third party logistics [D]. Jilin University, 2011.

[2] Zan army. Process reengineering of A logistics company vehicle shipping efficiency optimization research based on [D]. of Nanjing Agricultural University, 2013.

[3] Zhang Mengxia. Research on multi echelon inventory model based on stochastic demand and stochastic lead time [D]. Tsinghua University, 2012.

[4] Dai Jun, Xie Li, Jing Wong. The production enterprises of the third party logistics VMI of [J]. based on the modern management science, 2015 (1): 106-108.

[5] Hiassat A, Diabat A, Rahwan I. A genetic algorithm approach for location-inventory-routing problem with perishable products[J]. Journal of Manufacturing Systems, 2017, 42:93-103. 